\title{
PROYECCIONES CARTOGRÁFICAS
}

\section{$Y$ \\ SISTEMAS DE REFERENCIA}

Material de consulta de cátedra a partir de la compilación de bibliografía impresa y de Internet

TÉCNICAS EN GEOGRAFÍA I

Prof. Patricia Snaider 


\section{PROYECCIONES \\ CARTOGRAFICAS: definición}

Son transformaciones matemáticas que permiten representar (proyectar) a la esfera en el plano, y convertir las coordenadas geográficas (latitud \& longitud) en coordenadas cartesianas $(x \& y)$.

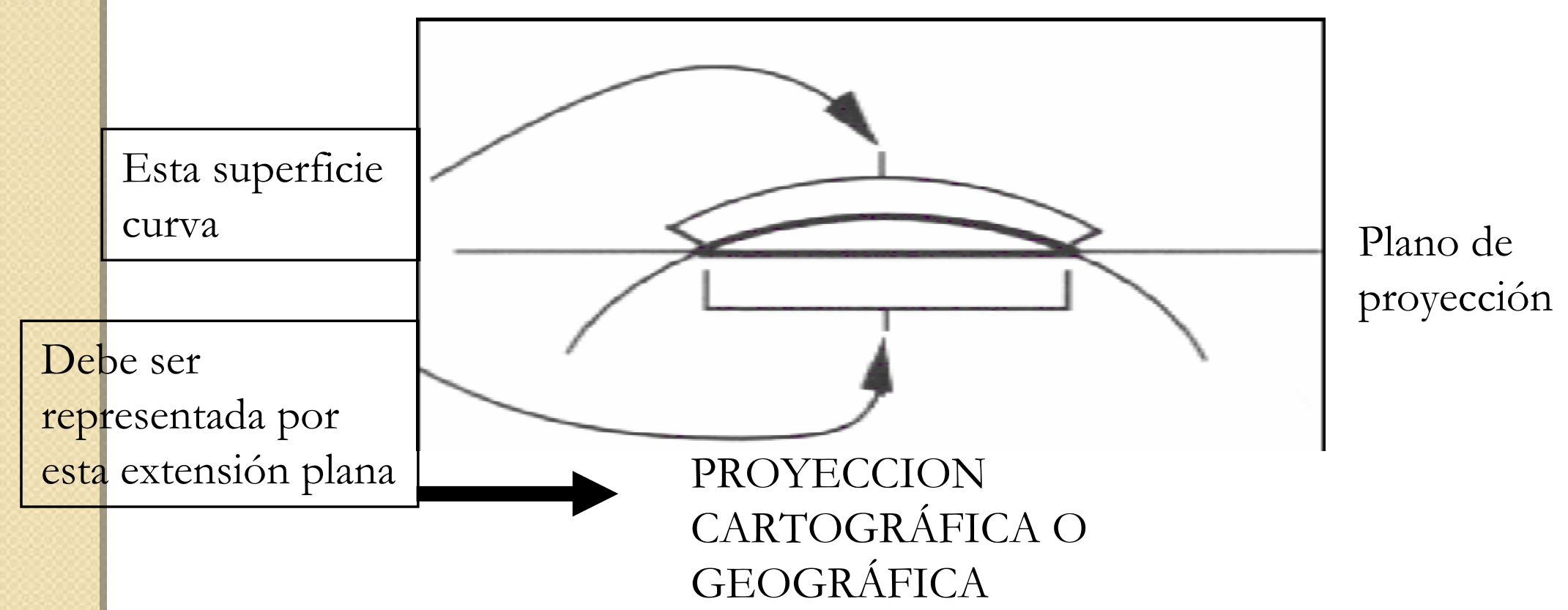




\section{PROYECCIONES}

\section{CARTOGRAFICAS: definición}

Este proceso conlleva distorsiones de la

superficie original en 3-dimensiones, al

convertirse a una superficie plana de dos dimensiones

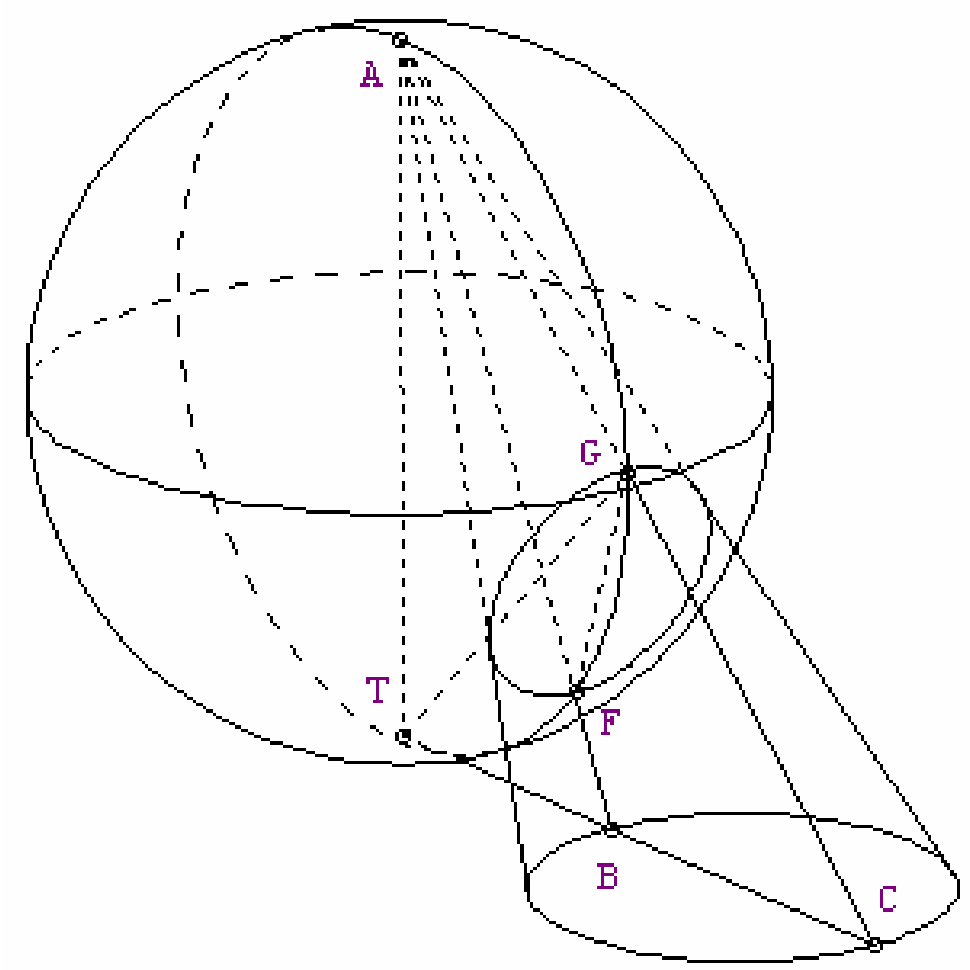




\section{PROYECCIONES \\ CARTOGRAFICAS: propiedades}

PROYECCIÓN CONFORME: conserva el ángulo entre dos puntos medidos en la superficie de referencia y en el mapa. Si no lo conserva se dice que la proyección tiene anamorfosis angular.

PROYECCIÓN EQUIDISTANTE: conserva las distancias; en todo el mapa no se cumple esta propiedad, pero debido a la escala, se puede considerar que las deformaciones son tan pequeñas que se admiten como tolerables. Cuando la proyección no cumple esta propiedad tiene anamorfosis lineal.

PROYECCIÓN EQUIVALENTE: conservan las superficies. Cuando no lo cumplen tienen anamorfosis superficial.

PROYECCIÓN AFILÁCTICA: no conservan ninguna de las propiedades anteriores pero tienen valores tolerables para determinadas zonas.

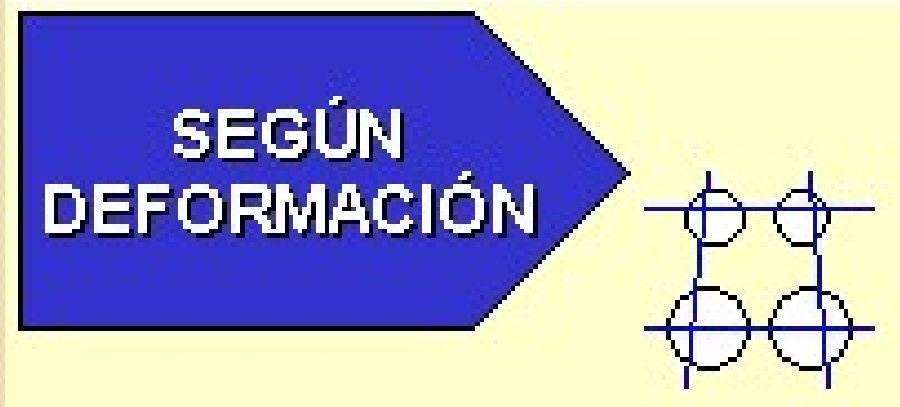

CONFOAME

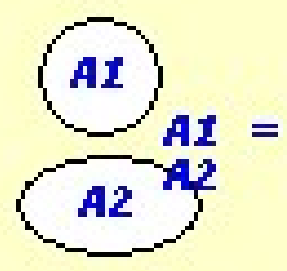

EQUTHALENTE

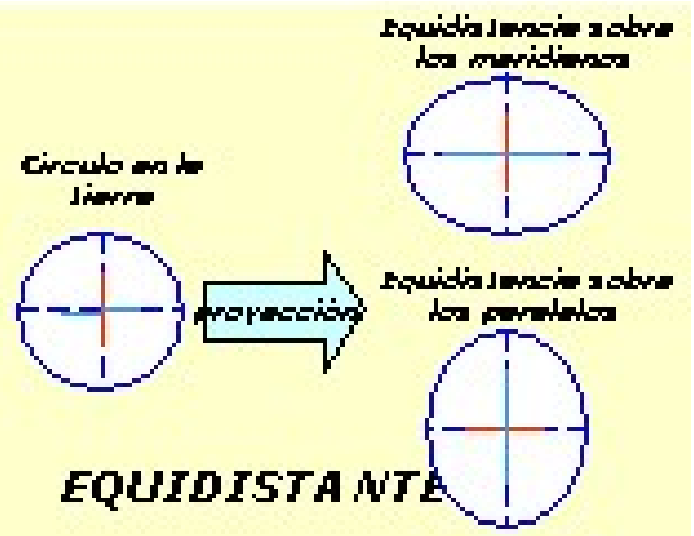

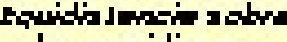
lo martion

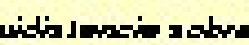




\section{PROYECCIONES}

\section{CARTOGRAFICAS: propiedades}

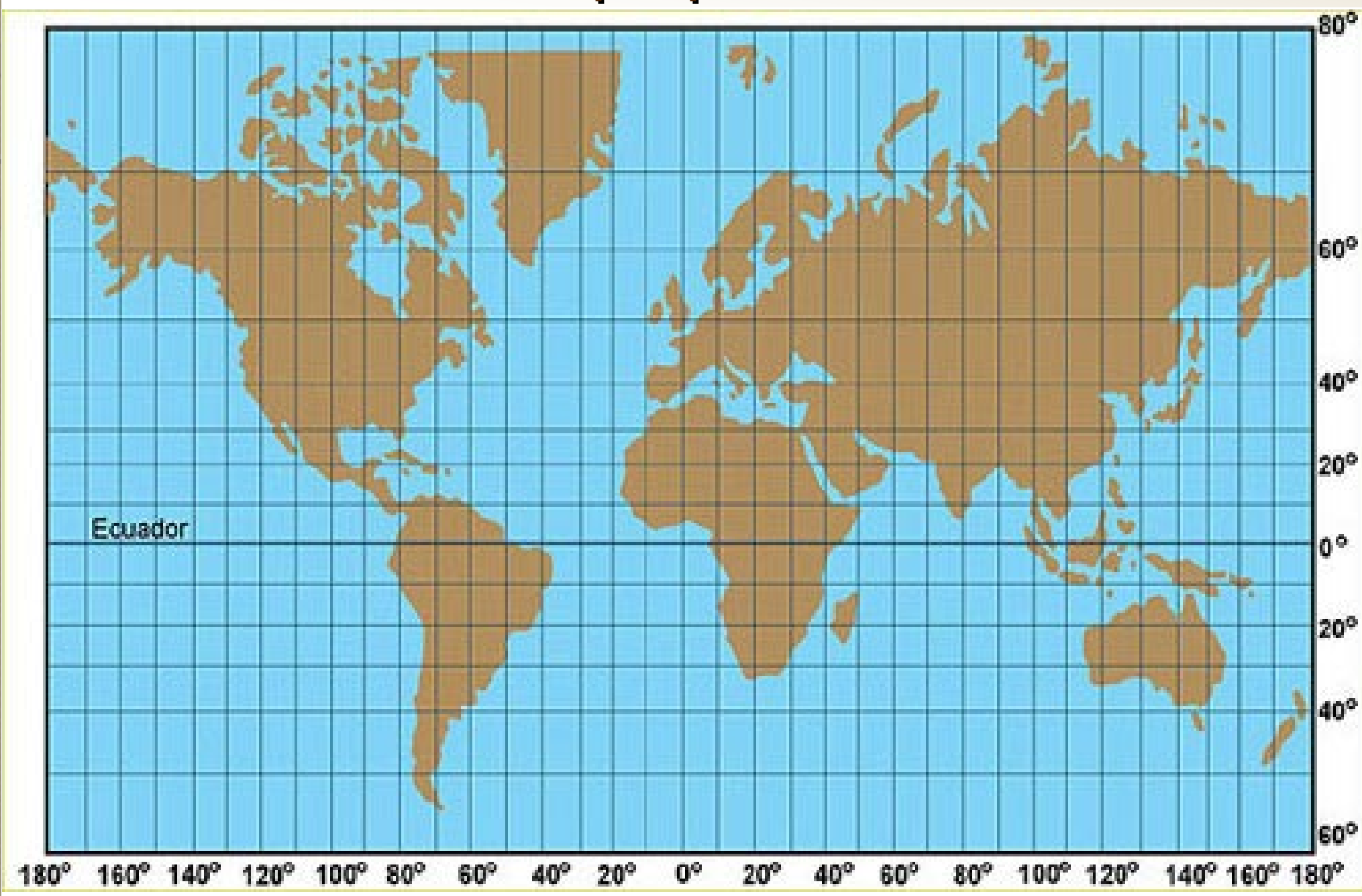

\section{PROYECCIÓN CONFORME}

Publicado en formato digital: Snaider, Patricia. PROYECCIONES CARTOGRÁFICAS Y SISTEMAS DE REFERENCIA. Resúmenes. Revista Geográfica Digital. IGUNNE. Facultad de Humanidades. UNNE. Año 7. № 13. Enero - Junio 2010. Resistencia, Chaco. En: http://hum.unne.edu.ar/revistas/geoweb/default.htm 


\section{CARTOGRAFICAS: propiedades}

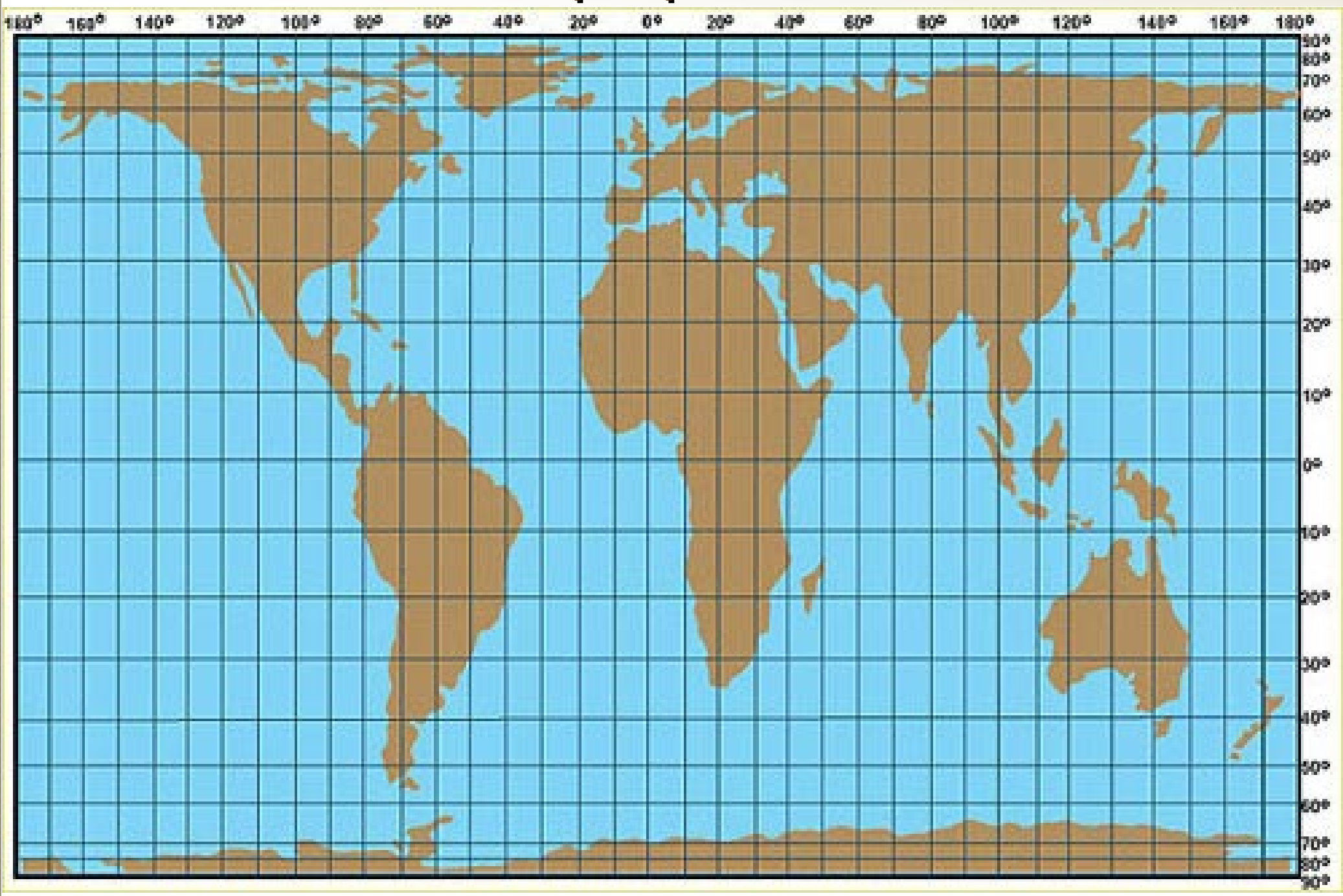

\section{PROYECCIÓN EQUIVALENTE}

Publicado en formato digital: Snaider, Patricia. PROYECCIONES CARTOGRÁFICAS Y SISTEMAS DE REFERENCIA. Resúmenes. Revista Geográfica Digital. IGUNNE. Facultad de Humanidades. UNNE. Año 7. № 13. Enero - Junio 2010. Resistencia, Chaco. En: http://hum.unne.edu.ar/revistas/geoweb/default.htm 


\section{PROYECCIONES}

\section{CARTOGRAFICAS: propiedades}

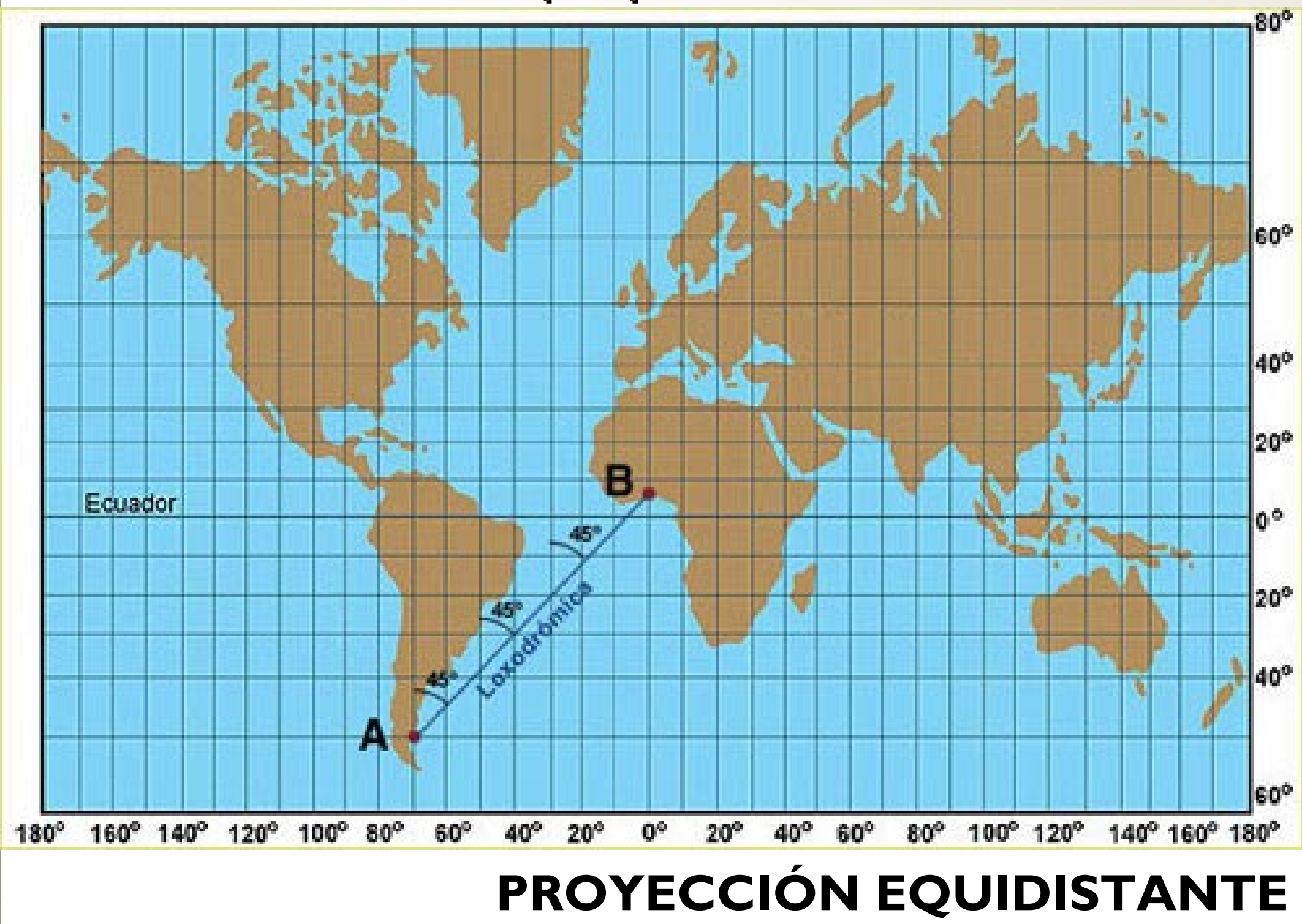

Publicado en formato digital: Snaider, Patricia. PROYECCIONES CARTOGRÁFICAS Y SISTEMAS DE REFERENCIA. Resúmenes. Revista Geográfica Digital. IGUNNE. Facultad de Humanidades. UNNE. Año 7. № 13. Enero - Junio 2010. Resistencia, Chaco. En: http://hum.unne.edu.ar/revistas/geoweb/default.htm 


\section{PROYECCIONES \\ CARTOGRAFICAS: clasificación}

\begin{tabular}{|c|c|c|}
\hline \multirow{2}{*}{$\begin{array}{l}\text { POR DESARROLLO (1 y 2) } \\
\text { Se proyecta la esfera sobre una } \\
\text { superficie desarrollable }\end{array}$} & \multicolumn{2}{|c|}{ CONICAS (1) } \\
\hline & \multicolumn{2}{|c|}{ CILINDRIDAS (2) } \\
\hline \multirow{4}{*}{$\begin{array}{l}\text { POR PERSPECTIVA O } \\
\text { ACIMUTALES (3) } \\
\text { Toda la superficie se proyecta sobre } \\
\text { un único plano de proyección. }\end{array}$} & \multirow{4}{*}{$\begin{array}{l}\mathrm{P} \\
\mathrm{L} \\
\mathrm{A} \\
\mathrm{N} \\
\mathrm{A} \\
\mathrm{S}\end{array}$} & ORTOGRÁFICAS \\
\hline & & ESCENOGRÁFICAS \\
\hline & & ESTEREOGRÁFICAS \\
\hline & & GNOMÓNICAS \\
\hline & & (3) \\
\hline
\end{tabular}

Publicado en formato digital: Snaider, Patricia. PROYECCIONES CARTOGRÁFICAS Y SISTEMAS DE REFERENCIA. Resúmenes. Revista Geográfica Digital. IGUNNE. Facultad de Humanidades. UNNE. Año 7. № 13. Enero - Junio 2010. Resistencia, Chaco. En: http://hum.unne.edu.ar/revistas/geoweb/default.htm 


\section{PROYECCIONES clasificación según tipo de CARTOGRAFICAS: superficie y orientación}

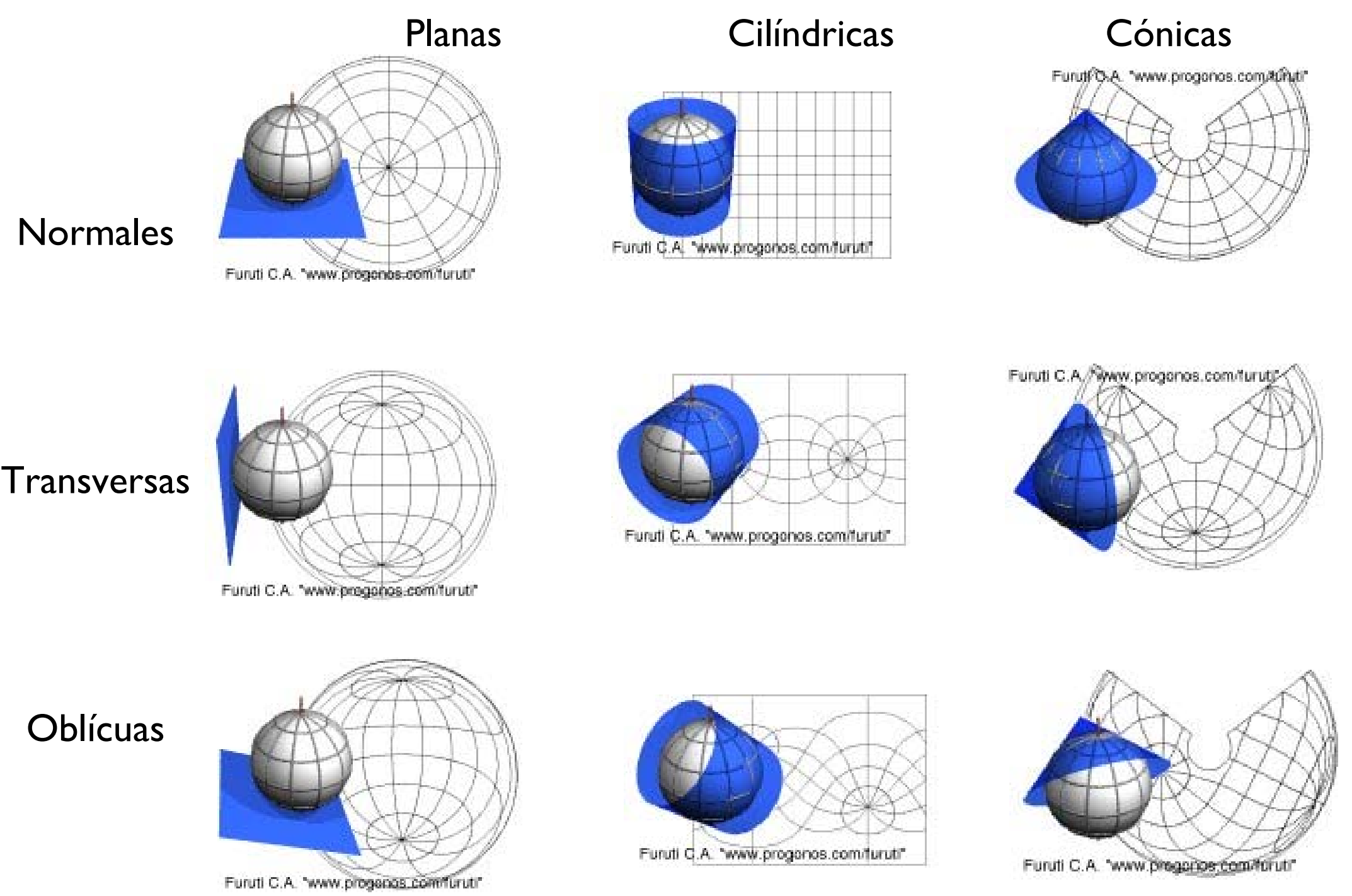

Publicado en formato digital: Snaider, Patricia. PROYECCIONES CARTOGRÁFICAS Y SISTEMAS DE REFERENCIA. Resúmenes. Revista Geográfica Digital. IGUNNE. Facultad de Humanidades. UNNE. Año 7. № 13. Enero - Junio 2010. Resistencia, Chaco. En: http://hum.unne.edu.ar/revistas/geoweb/default.htm 


\section{PROYECCIONES \\ CARTOGRAFICAS: cilíndrica}

- Proyección construida a partir de un cilindro: paralelos y meridianos son rectos.

- Permiten representar toda la superficie de la Tierra.

- El sector con menos deformación es la línea ecuatorial

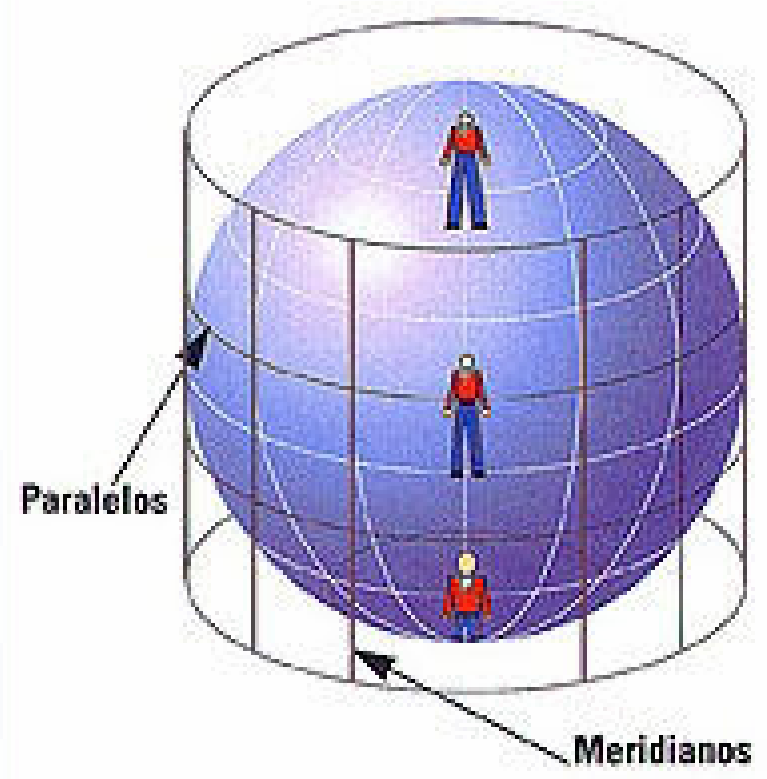

\section{PROYECCION CILINDRICA Meridianos Paralelos}

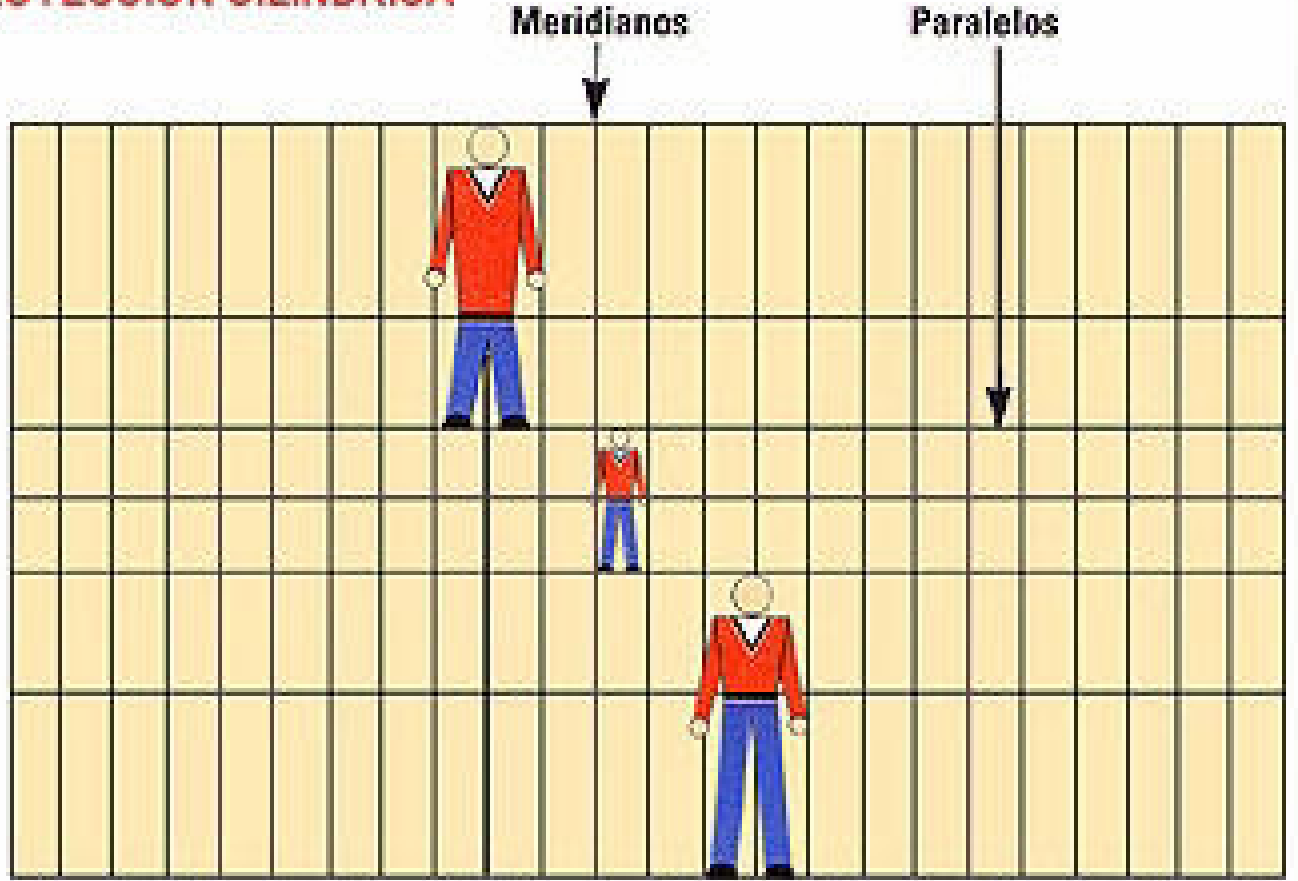

Publicado en formato digital: Snaider, Patricia. PROYECCIONES CARTOGRÁFICAS Y SISTEMAS DE REFERENCIA. Resúmenes. Revista Geográfica Digital. IGUNNE. Facultad de Humanidades. UNNE. Año 7. № 13. Enero - Junio 2010. Resistencia, Chaco. En: http://hum.unne.edu.ar/revistas/geoweb/default.htm 


\section{PROYECCIONES \\ CARTOGRAFICAS: cilindrica}

- La más famosa es la proyección de Mercator que revolucionó a la cartografía.

- En ella se proyecta el globo terrestre sobre un cilindro.

- Es una de las más utilizadas aun cuando por lo general en forma modificada, debido a las grandes distorsiones que ofrece en las zonas de latitud elevada, cosa que impide apreciar en sus verdaderas proporciones a las regiones polares.

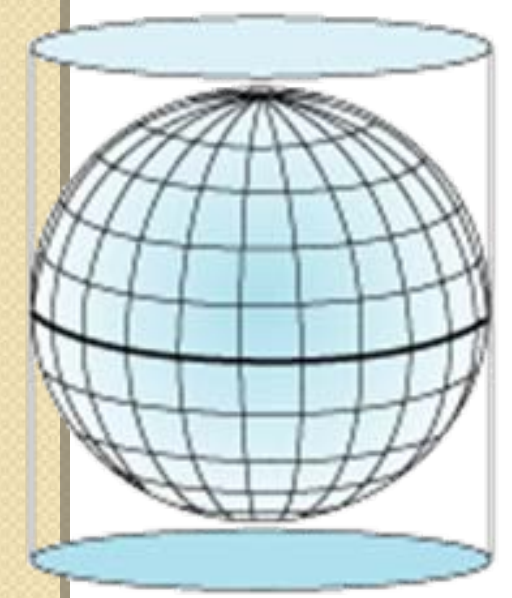

Normal

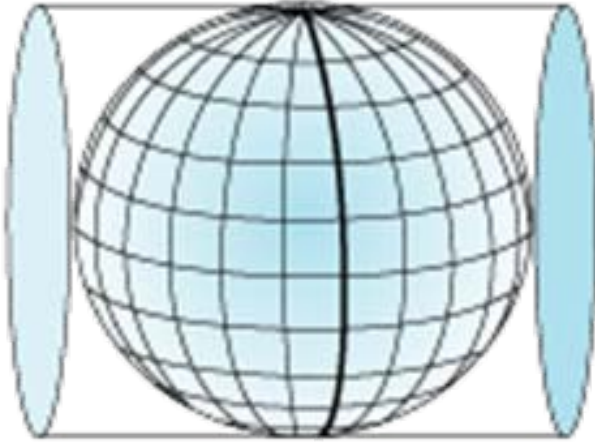

Transverse

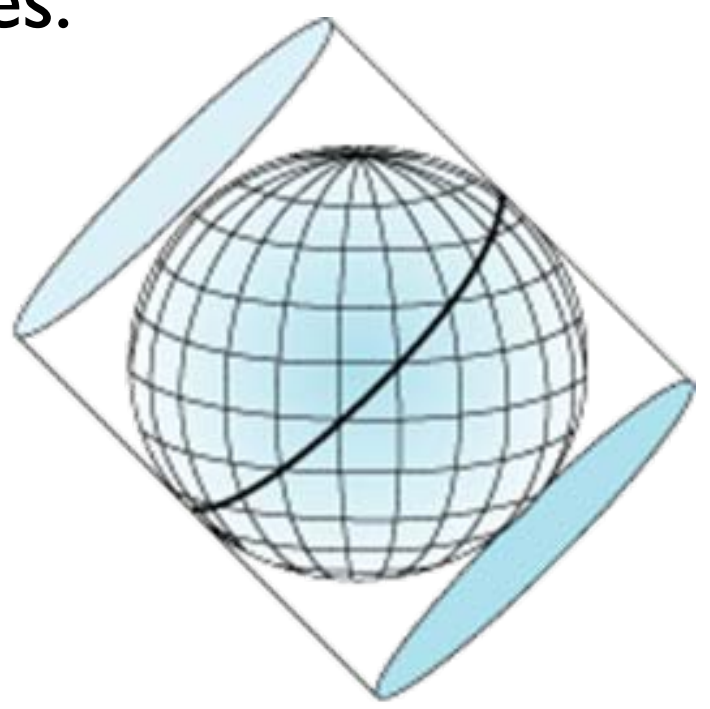

Oblique 


\section{PROYECCIONES \\ CARTOGRAFICAS: cónica}

- Proyección construida a partir de un cono: los meridianos se juntan en un punto y los paralelos son curvos.

- Es útil para representar latitudes medias. A lo largo del paralelo que toca el cono (tangente) se encuentra el sector con menos deformación.

\section{PROYECCIÓN CÓNICA}
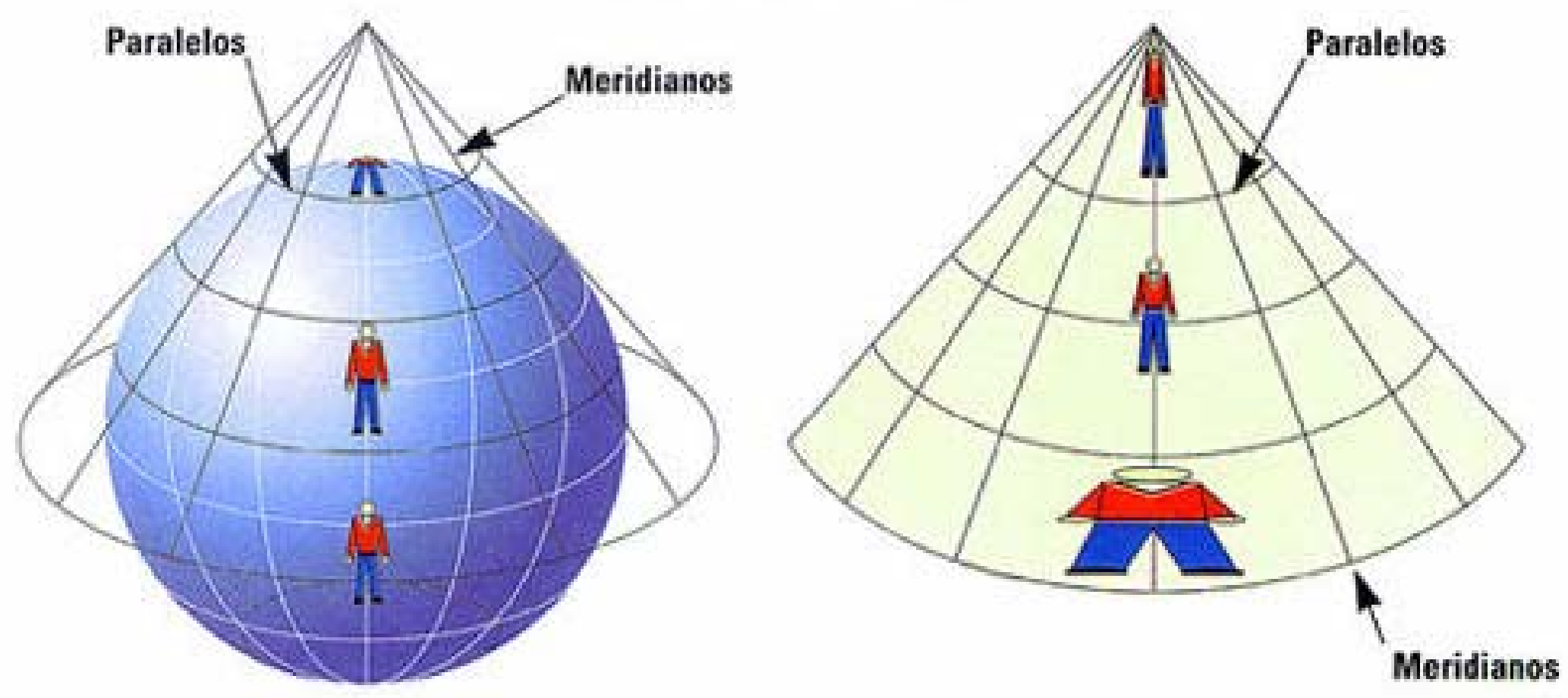

Publicado en formato digital: Snaider, Patricia. PROYECCIONES CARTOGRÁFICAS Y SISTEMAS DE REFERENCIA. Resúmenes. Revista Geográfica Digital. IGUNNE. Facultad de Humanidades. UNNE. Año 7. № 13. Enero - Junio 2010. Resistencia, Chaco. En: http://hum.unne.edu.ar/revistas/geoweb/default.htm 


\section{PROYECCIONES CARTOGRAFICAS: cónica}

Se origina una distorsión asimétrica que afecta, en gran medida, a las zonas polares, pero ofrece aceptable precisión en las zonas del hemisferio donde el cono de proyección es tangente.

- La imagen proyectada en la superficie cónica se "despliega", resultando un dibujo plano, de fácil reproducción en una hoja de papel.

- Se utiliza, preferentemente, para representar aquellos países que se encuentran en las regiones de latitudes medias, por ser menor la distorsión resultante. 


\section{PROYECCIONES}

\section{CARTOGRAFICAS: cónica}

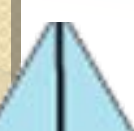

\section{Standard parallel}

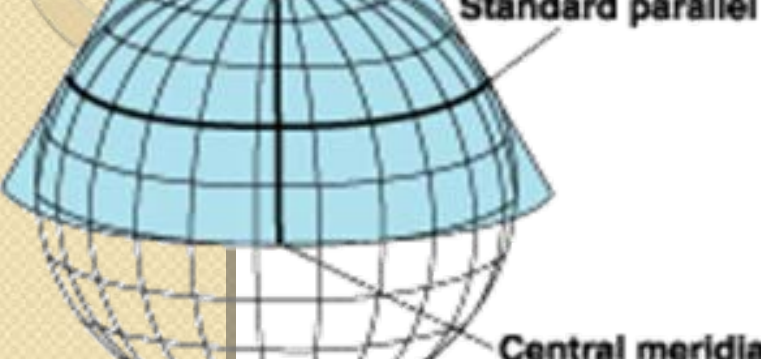

Central meridian

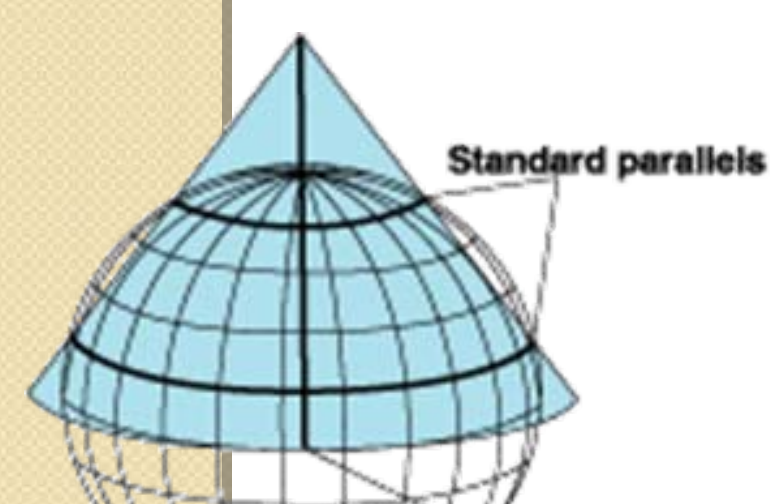

Central meridian
TANGENTE
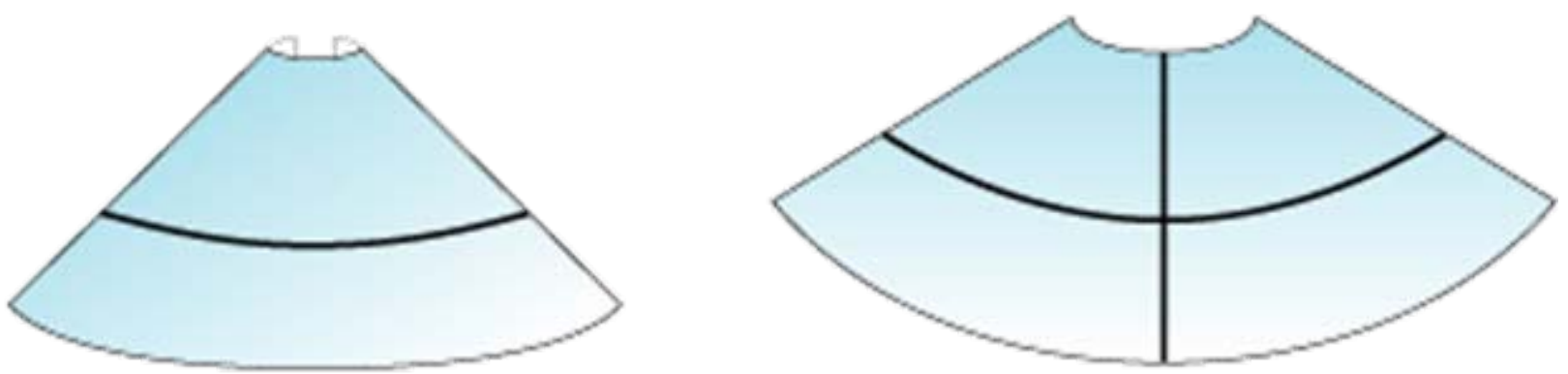

SECANTE
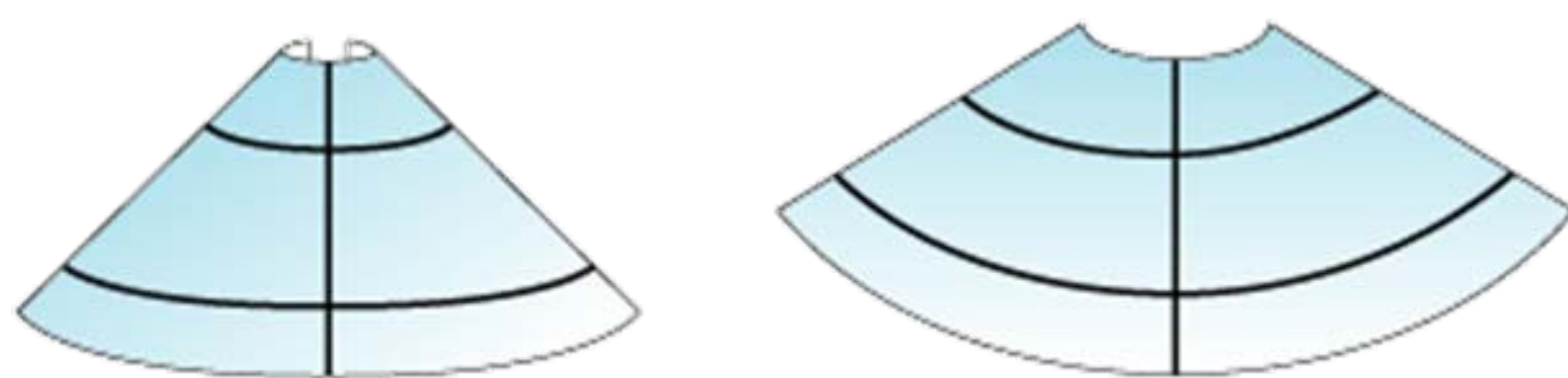

Publicado en formato digital: Snaider, Patricia. PROYECCIONES CARTOGRÁFICAS Y SISTEMAS DE REFERENCIA. Resúmenes. Revista Geográfica Digital. IGUNNE. Facultad de Humanidades. UNNE. Año 7. № 13. Enero - Junio 2010. Resistencia, Chaco. En: http://hum.unne.edu.ar/revistas/geoweb/default.htm 


\section{PROYECCIONES \\ CARTOGRAFICAS: plana}

- Construida a partir de un plano. Representan un hemisferio y su línea externa es un círculo.

\section{PROYECCIÓN PLAMA POLAR}
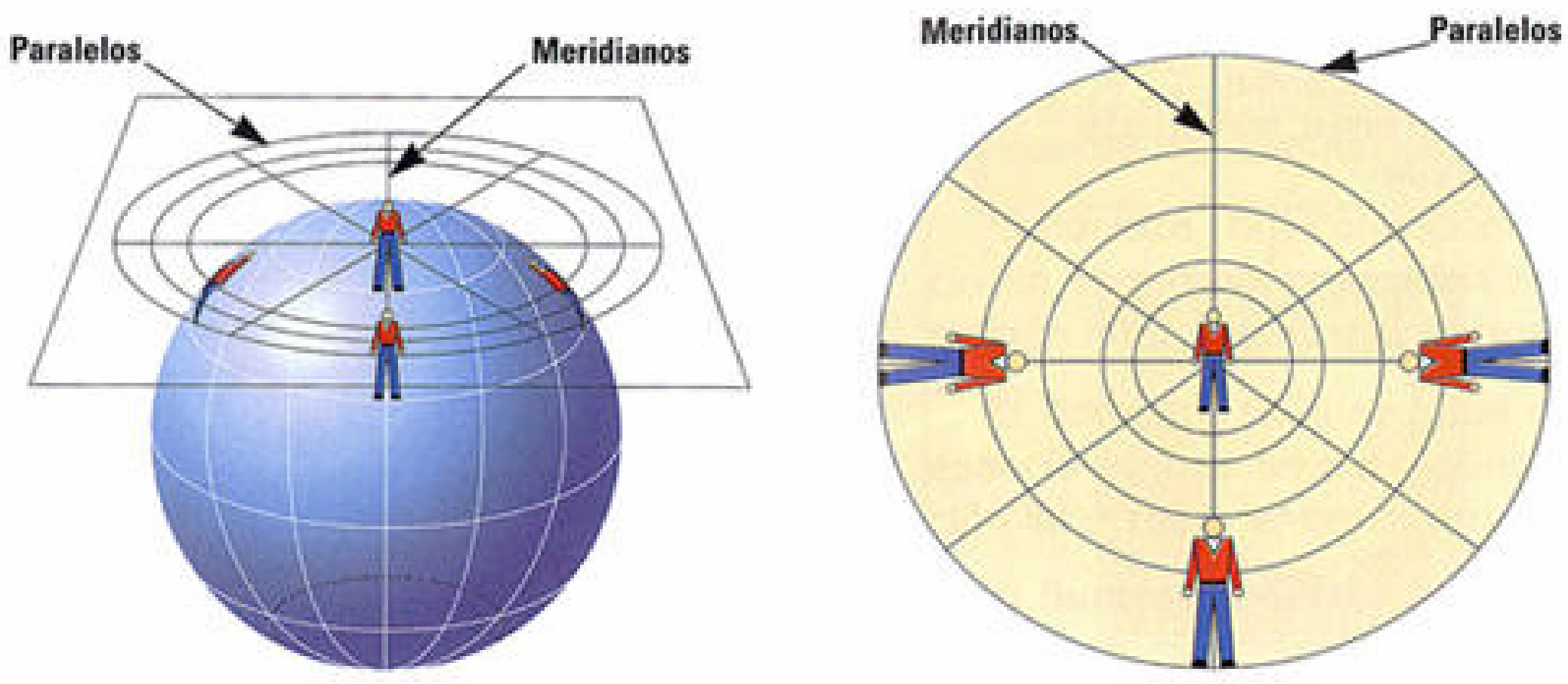

Publicado en formato digital: Snaider, Patricia. PROYECCIONES CARTOGRÁFICAS Y SISTEMAS DE REFERENCIA. Resúmenes. Revista Geográfica Digital. IGUNNE. Facultad de Humanidades. UNNE. Año 7. № 13. Enero - Junio 2010. Resistencia, Chaco. En: http://hum.unne.edu.ar/revistas/geoweb/default.htm 


\section{PROYECCIONES \\ CARTOGRAFICAS: plana}

- Pueden ser polares, si uno de los polos está en el centro de la proyección; el sector más preciso es alrededor del polo.

- También pueden ser ecuatoriales cuando un punto de la línea ecuatorial ocupa el centro de la proyección y, en las oblicuas, el centro corresponde a un punto intermedio, entre un polo y el ecuador

- O pueden ser oblicuas cuando el centro corresponde a un punto intermedio, entre un polo y el ecuador
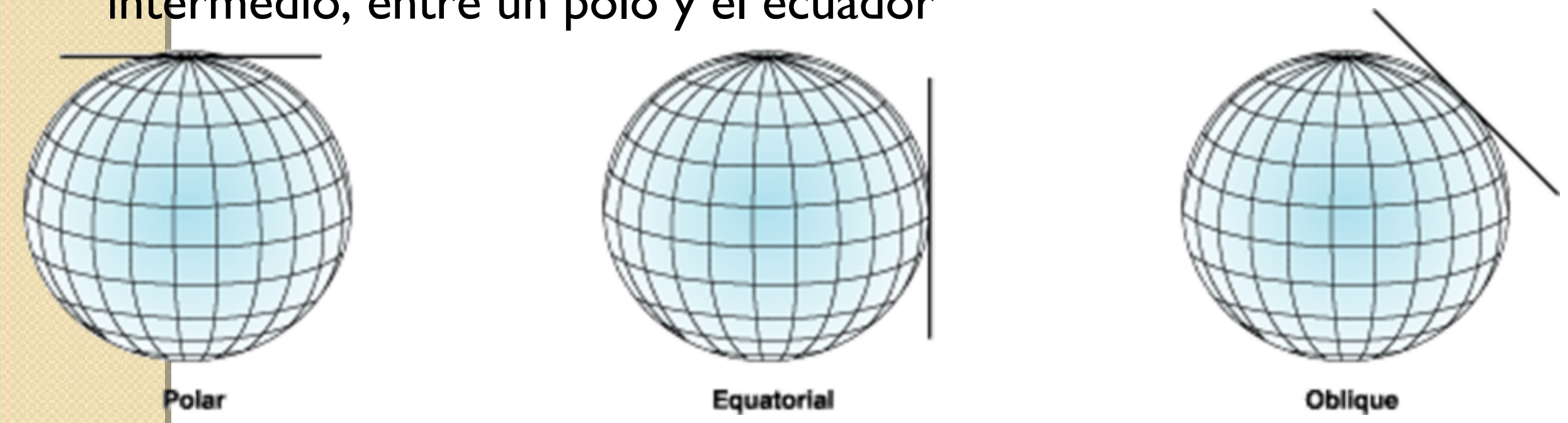

Publicado en formato digital: Snaider, Patricia. PROYECCIONES CARTOGRÁFICAS Y SISTEMAS DE REFERENCIA. Resúmenes. Revista Geográfica Digital. IGUNNE. Facultad de Humanidades. UNNE. Año 7. № 13. Enero - Junio 2010. Resistencia, Chaco. En: http://hum.unne.edu.ar/revistas/geoweb/default.htm 


\section{PROYECCIONES CARTOGRAFICAS: plana}

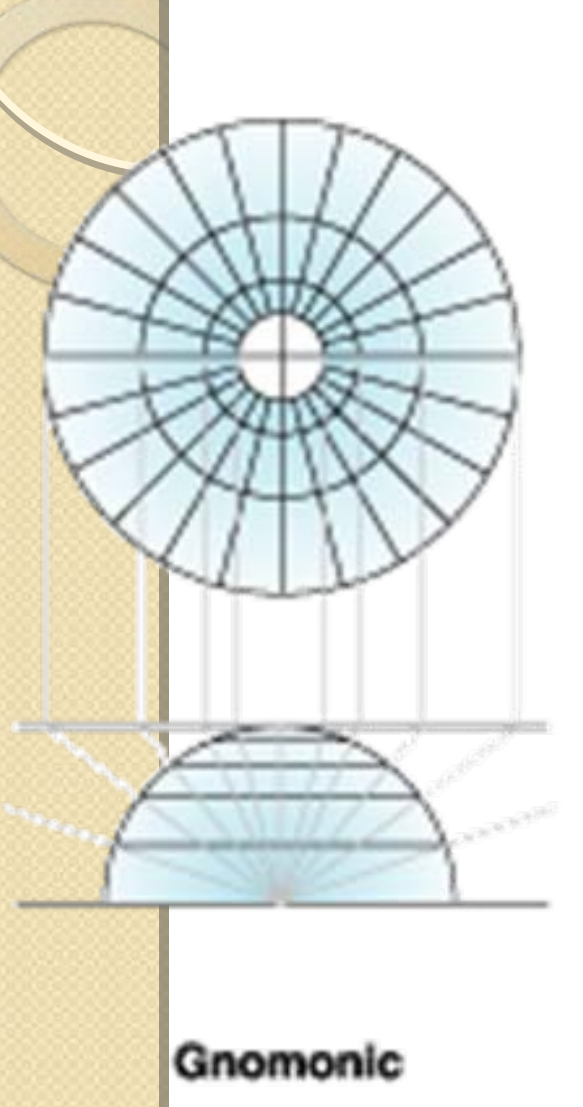

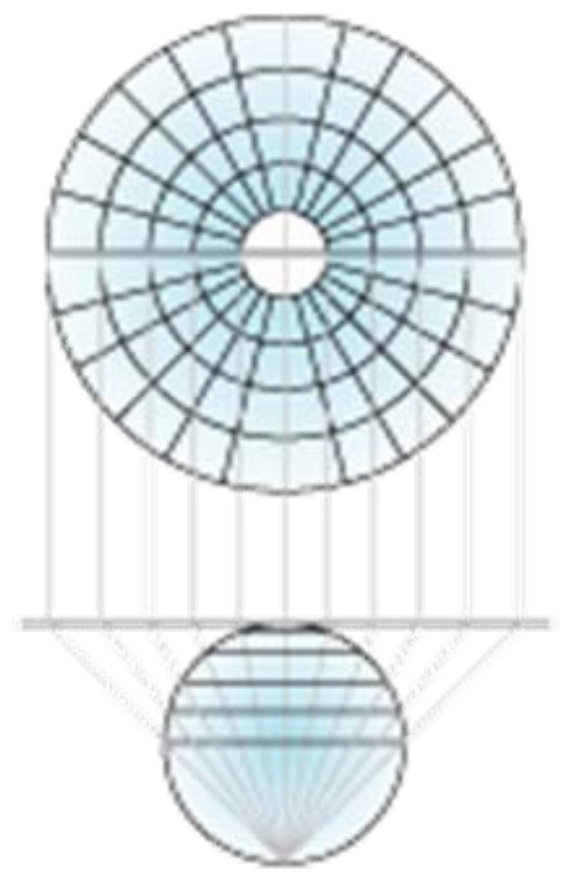

Stereographic

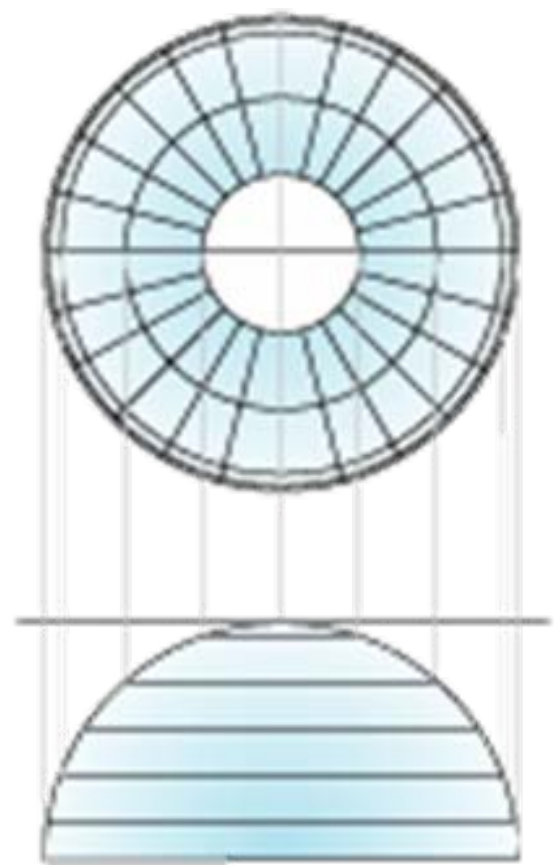

Orthographic 


\section{PROYECCIONES Proyección Argentina CARTOGRAFICAS: Gauss-Krüger}

- El I.G.M. ha adoptado para la cartografía oficial el sistema de proyección GaussKrüger.

- Se trata de una proyección conforme, cilíndrica y transversal.

- La primera propiedad permite representar los objetos espaciales conservando las formas.

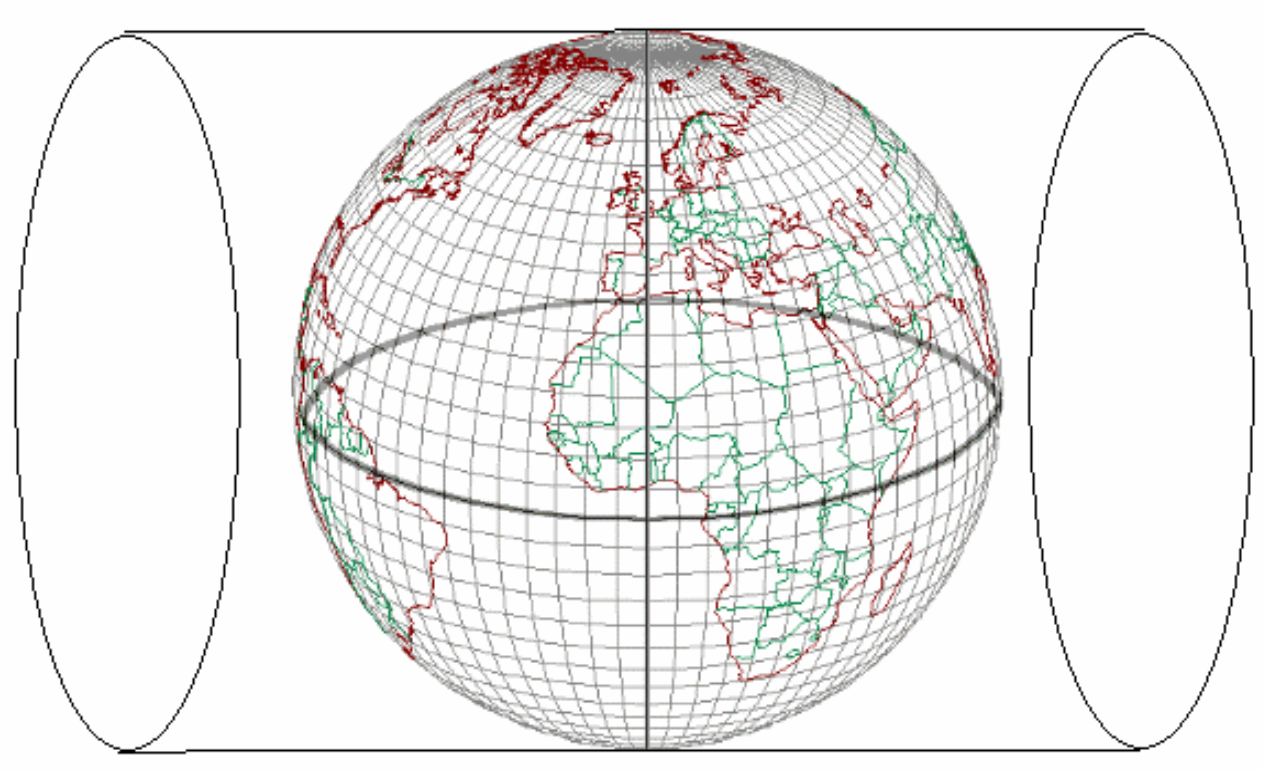

- La transferencia analítica del elipsoide al plano se efectúa sobre un cilindro tangente $a$ un meridiano. 


\section{PROYECCIONES}

CARTOGRAFICAS: Gauss-Krüger

\begin{tabular}{|c|c|c|c|c|c|c|c|}
\hline Faja & 1 & 2 & 3 & 4 & 5 & 6 & 7 \\
\hline \hline bordes & $73.5^{\circ}$ & $70.5^{\circ}$ & $67.5^{\circ}$ & $64.5^{\circ}$ & $61.5^{\circ}$ & $58.5^{\circ}$ & $55.5^{\circ}$ \\
& $70.5^{\circ}$ & $67.5^{\circ}$ & $64.5^{\circ}$ & $61.5^{\circ}$ & $58.5^{\circ}$ & $55.5^{\circ}$ & $52.5^{\circ}$ \\
\hline $\begin{array}{c}\text { meridiano } \\
\text { central }\end{array}$ & $72^{\circ}$ & $69^{\circ}$ & $66^{\circ}$ & $63^{\circ}$ & $60^{\circ}$ & $57^{\circ}$ & $54^{\circ}$ \\
\hline
\end{tabular}

- Factor de escala en el meridiano central $=\mathrm{I}$.

- La adopción de 7 fajas de $3^{\circ}$ de ancho obedece a la necesidad de reducir las deformaciones en los bordes.

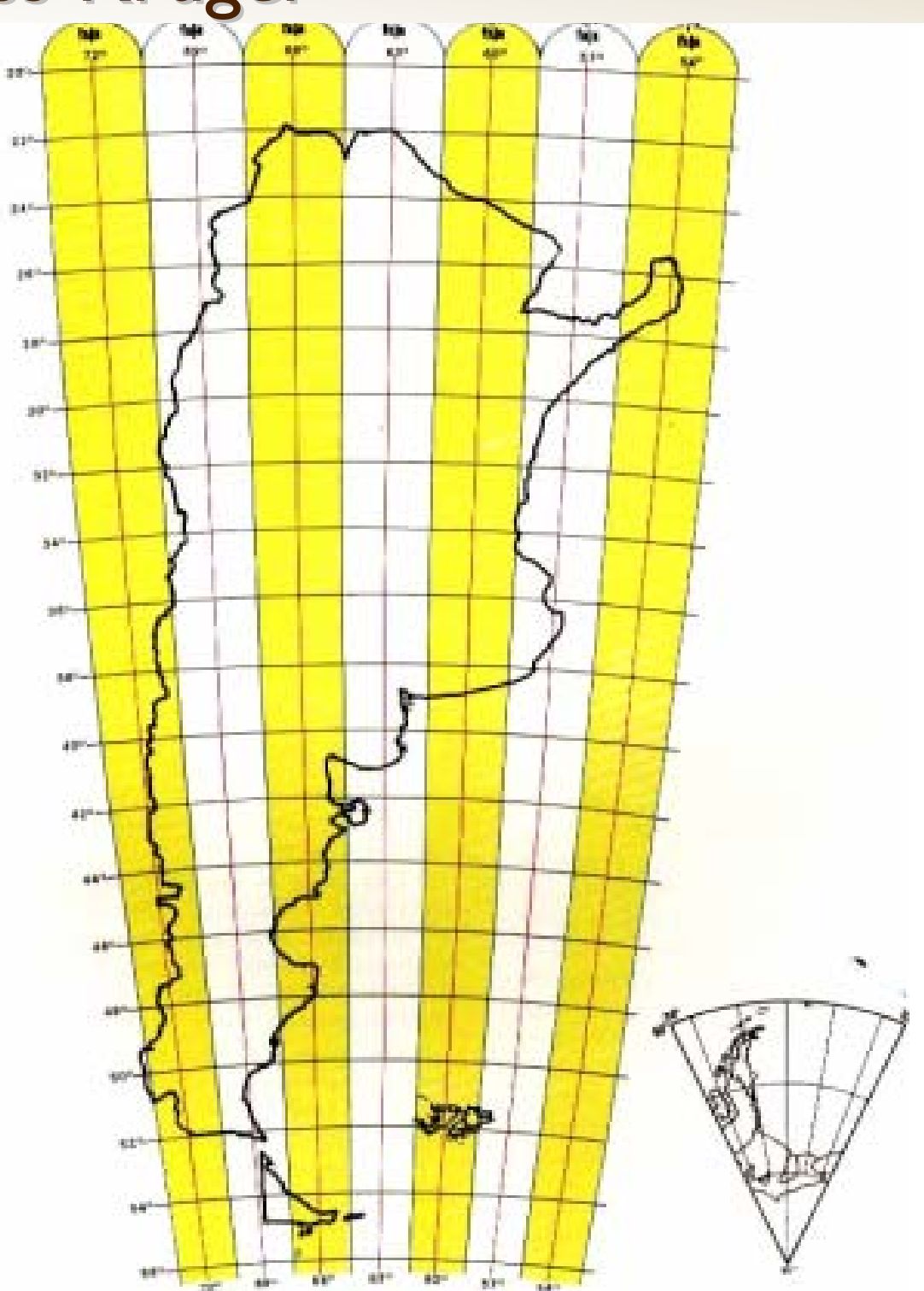




\section{SISTEMAS DE REFERENCIA: definición}

Un sistema de referencia geodésico es un recurso matemático que permite asignar coordenadas a puntos sobre la superficie terrestre.

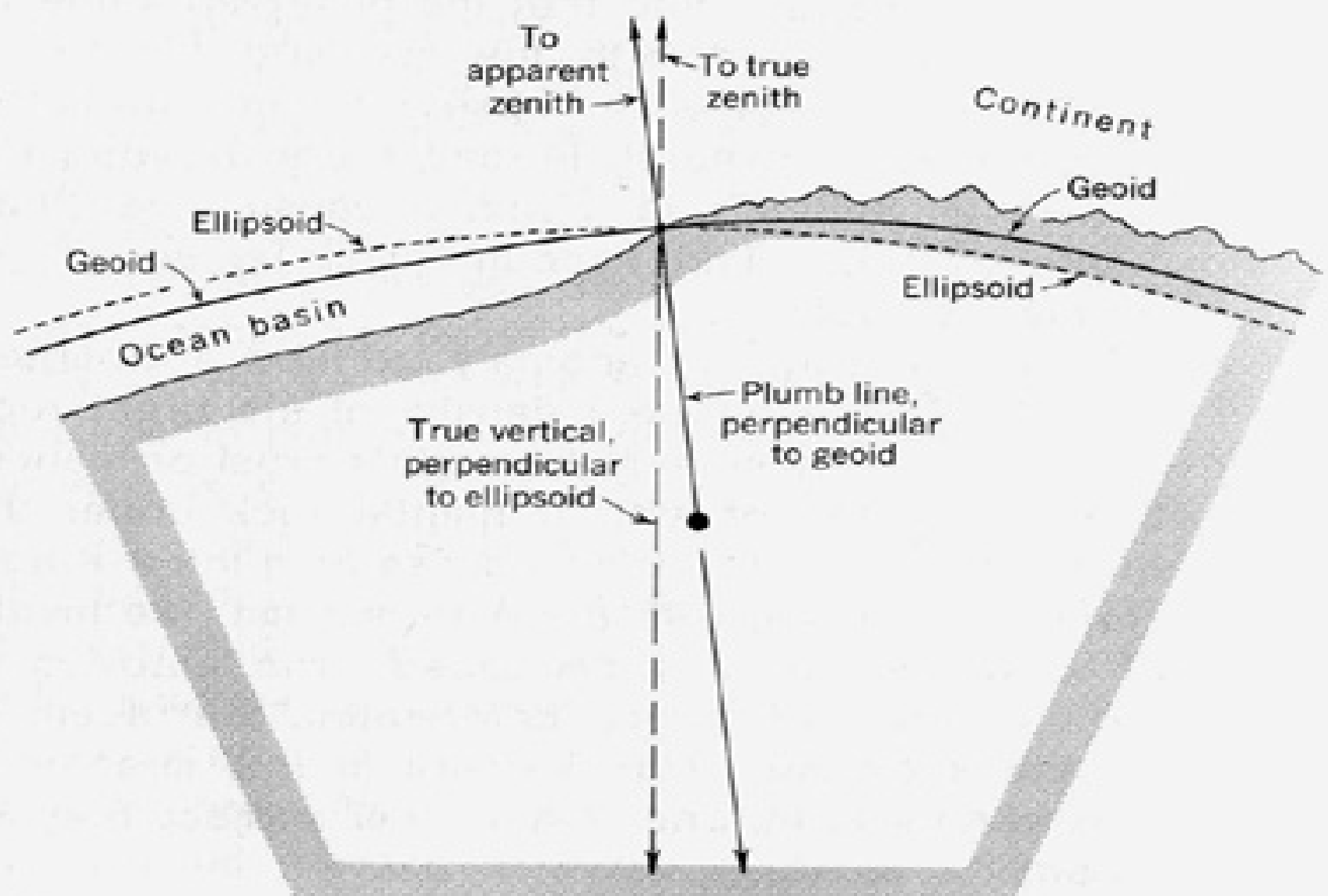




\section{SISTEMAS DE REFERENCIA: componentes}

- ELIPSOIDE: producto de la rotación (achatamiento polos), difiere ligeramente de la esfera

- GEOIDE: superficie gravimétrica, difiere ligeramente del elipsoide (la gravedad varía con el relieve superficial y submarino)

- DATUM: define sistemas de referencia que describen el tamaño y la forma de la Tierra (Geodesia) 


\section{SISTEMAS DE REFERENCIA: locales y globales}

LOCALES: utilizan para su definición un elipsoide determinado y un punto datum.

- RAMSAC (Red Argentina de Monitoreo Satelital Continuo) son estaciones GPS permanentes o activas

GLOBALES: cuyos parámetros están dados por una terna rectangular $(X, Y, Z)$ cuyo origen se encuentra en el geocentro del Planeta.

- WGS 84 (World Geocentric System) 


\section{MARCOS DE REFERENCIA GEODÉSICOS}

- Constituyen la materialización del sistema de referencia sobre la superficie terrestre.

- Un sistema de referencia se materializa mediante un conjunto de mojones geodésicos implantados en una región, a los que se le han asignado coordenadas.

- Son las redes de puntos de los Sistemas de Referencia. 


\section{MARCOS DE REFERENCIA MUNDIAL}

- IRTF (International Terrestrial Reference Frame Marco de Referencia Terrestre Internacional)

\section{MARCOS DE REFERENCIA GEODÉSICOS}

- Campo Inchauspe 1969

- POSGAR 94

- POSGAR 98

- POSGAR 2007

- SIRGAS (Marco de Referencia Geocéntrico para las Américas) red sudamericana de muy alta precisión 